Service social

\title{
Connaissances et utilisation des méthodes contraceptives et de la contraception d'urgence par les étudiantes québécoises de niveau collégial et universitaire : résultats de deux enquêtes complémentaires
}

\author{
Sylvie Lévesque, Sara Mathieu-C., Édith Guilbert, Gilles Lambert, Dominic \\ Beaulieu Prévost, Martin Blais, Marie-Aude Boislard et Joseph Lévy
}

Volume 63, numéro 2, 2017

Genres et sexualités chez les jeunes

URI : https://id.erudit.org/iderudit/1046499ar

DOI : https://doi.org/10.7202/1046499ar

Aller au sommaire du numéro

Éditeur(s)

École de travail social et de criminologie de l’Université Laval

ISSN

1708-1734 (numérique)

Découvrir la revue

Citer cet article

Lévesque, S., Mathieu-C., S., Guilbert, É., Lambert, G., Beaulieu Prévost, D., Blais, M., Boislard, M.-A. \& Lévy, J. (2017). Connaissances et utilisation des méthodes contraceptives et de la contraception d'urgence par les étudiantes québécoises de niveau collégial et universitaire : résultats de deux enquêtes

complémentaires. Service social, 63(2), 50-70. https://doi.org/10.7202/1046499ar

\section{Résumé de l'article}

L'objectif de cet article est de présenter une mise à jour sur l'utilisation des méthodes contraceptives, y compris la contraception d'urgence, chez les femmes sexuellement actives qui fréquentent les cégeps et les universités du Québec. Cet article puise dans deux études sur la santé sexuelle menées en milieu collégial et universitaire (ESS-UQAM et PIXEL). Les principaux constats de ces études réalisées auprès d'un échantillon de 1812 universitaires (ESS-UQAM) et d'un échantillon de 921 étudiantes recrutées dans 17 cégeps et 7 universités (PIXEL) révèlent que, globalement, la proportion d'étudiantes québécoises utilisant des méthodes contraceptives efficaces est élevée et que la non-utilisation de contraception est faible (entre 2,9 \% et $6 \%$ ). L'utilisation de méthodes contraceptives semble varier selon le parcours migratoire et l'orientation sexuelle des étudiantes. Ces résultats sont discutés dans la perspective de faire émerger certains des enjeux actuels en matière de contraception et d'orienter la prise de décision à l'égard d'interventions préventives. 


\title{
Connaissances et utilisation des méthodes contraceptives et de la contraception d'urgence par les étudiantes québécoises de niveau collégial et universitaire : résultats de deux enquêtes complémentaires
}

\author{
LÉVESQUE, Sylvie, Ph.D. \\ MATHIEU-C., Sara, Ph.D.(c) \\ GUILBERT, Édith, M.D., M.Sc. \\ LAMBERT, Gilles, M.D.
}

Équipe ESS-UQAM : BEAULIEU PRÉVOST, Dominic, Ph.D., BLAIS, Martin, Ph.D., BOISLARD, Marie-Aude, Ph.D., LÉVY, Joseph, Ph.D.

\begin{abstract}
RÉSUMÉ
L'objectif de cet article est de présenter une mise à jour sur l'utilisation des méthodes contraceptives, y compris la contraception d'urgence, chez les femmes sexuellement actives qui fréquentent les cégeps et les universités du Québec. Cet article puise dans deux études sur la santé sexuelle menées en milieu collégial et universitaire (ESS-UQAM et PIXEL). Les principaux constats de ces études réalisées auprès d'un échantillon de 1812 universitaires (ESS-UQAM) et d'un échantillon de 921 étudiantes recrutées dans 17 cégeps et 7 universités (PIXEL) révèlent que, globalement, la proportion d'étudiantes québécoises utilisant des méthodes contraceptives efficaces est élevée et que la non-utilisation de contraception est faible (entre 2,9\% et $6 \%$ ). L'utilisation de méthodes contraceptives semble varier selon le parcours migratoire et l'orientation sexuelle des étudiantes. Ces résultats sont discutés dans la perspective de faire émerger certains des enjeux actuels en matière de contraception et d'orienter la prise de décision à l'égard d'interventions préventives.
\end{abstract}

Mots clés : Contraception, cégep, université, jeunes femmes, contraception d'urgence

\begin{abstract}
This paper presents an update on the uses of contraceptive methods among young sexually active women attending college or university in Quebec. Results, drawn from two surveys of young adults' sexual health, 1,812 university students (UQAM Study) and 921 students recruited in 17 colleges and 7 universities (PIXEL study), reveal that overall, contraceptive use rates in Quebec are high and the proportion of students who do not use contraception is low, although there are some variability according to migratory pattern and sexual orientation. These findings are discussed with the aim of highlighting some of the current contraceptive issues and guiding decision-making about preventive interventions.
\end{abstract}

Keywords: Contraception, College, University, Young Women, Emergency Contraception 


\section{Contexte}

Au Canada, on estime que les grossesses non planifiées représentent $40 \%$ de toutes les grossesses (Black et al., 2015b). Selon une enquête menée par l'Agence de santé publique du Canada (2009), $20,0 \%$ des mères canadiennes auraient préféré concevoir plus tard et 7,1\% pas du tout ; et cela dans des proportions significativement plus élevées chez les mères âgées de moins de 25 ans comparativement aux mères plus âgées. Au Québec, la proportion de grossesses non planifiées est inconnue, mais la baisse des interruptions volontaires de grossesse (IVG) couplée à la baisse du taux de fécondité chez les moins de 25 ans au cours des quinze dernières années laisse supposer un déclin de la proportion des grossesses non planifiées (Black et al., 2015b). De 2000 à 2014, le taux d'IVG est passé de 22,3/1000 à 13,6/1000 chez les femmes de 15 à 19 ans et de 35,2/1000 à 27,0/1000 chez celles de 20 à 24 ans (Institut de la statistique du Québec, 2017a). Pour sa part, le taux de fécondité a diminué de 13,3/1000 à 6,8/1000 chez les femmes de 15 à 19 ans et de 60,0/1000 à 38,9/1000 chez celles de 20 à 24 ans de 2000 à 2015 (Institut de la statistique du Québec, 2017b).

Une proportion importante des des femmes âgées de moins de 25 ans sont des étudiantes au cégep ou à l'université qui souhaitent terminer leurs études avant de devenir mères (Tyden et al., 2006 ; Virtala et al., 2006). Pour plusieurs jeunes femmes, s'occuper d'une grossesse non planifiée menace la capacité de poursuivre des études en raison des coûts liés à la parentalité (par ex. logement, services de garde) et d'un accès aux prêts étudiants peu adaptés (Prentice, Storin et Robinson, 2012). Comme ces étudiantes font partie d'un groupe dont une forte majorité indique être sexuellement active (Rotermann et McKay, 2009 ; Joubert et Du Mays, 2014), la prévention des grossesses non planifiées est un enjeu social et de santé prioritaire.

L'utilisation efficace des méthodes contraceptives et le recours en temps opportun à la contraception d'urgence $(\mathrm{CU})$ peuvent contribuer à la prévention des grossesses non planifiées et au maintien de la santé sexuelle. L'accès à ces méthodes contraceptives peut toutefois s'avérer inégal selon le groupe ethnoculturel, le statut relationnel, l'orientation sexuelle ou le statut socio-économique des jeunes femmes (Centers for Disease Control and Prevention, 2012 ; Dehlendorf et al., 2010 ; Hartnett, Lindley et Walsemann, 2017 ; Janevic et al., 2012 ; Mosher et Jones, 2010).

Au Québec, depuis 1997 et dans la majorité des cas, la contraception hormonale $(\mathrm{CH})$ et la CU sont disponibles gratuitement et sans prescription médicale aux femmes de moins de 18 ans et aux étudiantes à temps plein âgées de 25 ans et moins résidant chez leurs parents (LegisQuebec, 2002). Des initiatives novatrices ont également été implantées dans les dernières années pour faciliter l'accès à la contraception (Guilbert et al., 2013). Parmi celles-ci, notons une stratégie de partage de tâches et d'élargissement des rôles permettant aux infirmières et aux pharmaciens de prescrire la contraception hormonale (Guilbert et al., 2014). Les cliniques jeunesse des CLSC et les services de santé en milieu scolaire ont bénéficié de cet élargissement des actes réservés.

Considérant le manque de données provinciales sur le recours à la contraception, deux équipes de recherche ont mené, en 2013-2014, des enquêtes parallèles sur la santé sexuelle d'étudiant-e-s québécois-e-s. L'objectif de cet article est de présenter un état de situation de l'utilisation des méthodes contraceptives, y compris la contraception d'urgence, chez les femmes sexuellement actives qui fréquentent les cégeps et les universités du Québec. 


\section{MÉTHODOLOGIE}

Les deux enquêtes menées auprès d'étudiant-e-s québécois-e-s au cégep ou à l'université ont été réalisées, l'une par le département de sexologie de l'Université du Québec à Montréal (Enquête sur la santé sexuelle des étudiants de l'UQAM), et l'autre par l'Institut national de santé publique du Québec (Étude PIXEL - Portrait de la santé sexuelle des jeunes adultes québécois).

\section{Enquête sur la santé sexuelle des étudiants de I'UQAM (ESS-UQAM)}

Procédures. La collecte de données s'est effectuée entre octobre et novembre 2013 auprès de 3115 étudiant-e-s de l'UQAM. Les participant-e-s ont répondu à un questionnaire en ligne multidimensionnel. Les étudiant-e-s inscrit-e-s à l'automne 2013 à l'UQAM ont reçu des informations sur l'enquête par courrier électronique et ont été invité-e-s à y participer. Les participant-e-s éligibles ont donné leur consentement électronique avant d'accéder au sondage. Les réponses ont été entreposées sur un serveur sécurisé. Le Comité institutionnel d'éthique de la recherche avec des êtres humains de l'UQAM a approuvé l'enquête.

Instrument de mesure. Le questionnaire, élaboré à la suite d'une consultation d'experts dans le domaine de la sexologie ainsi que d'un examen approfondi des écrits de la littérature, comportait diverses mesures explorant 11 dimensions de la santé sexuelle: habitudes de vie; activités, pratiques et expériences sexuelles ; vie universitaire et sexuelle ; infections transmissibles sexuellement et par le sang (ITSS) et protection; contraception et IVG ; genre et orientation sexuelle ; fonctionnement et satisfaction sexuels ; valeurs et attitudes ; victimisation, intimidation et discrimination ; relation au corps ; santé et bien-être. II s'y trouvait également une section sur les besoins d'informations sur la sexualité. Le questionnaire a été prétesté avec un groupe mixte de 6 étudiant-e-s universitaires. Lors de l'enquête, les participant-e-s ont aussi été interrogé-e-s sur leur âge, la fréquentation d'institutions ou de services religieux, leur trajectoire migratoire (né-e à l'extérieur du Canada ou parents nés à l'extérieur du Canada), leurs expériences de difficultés financières au moment de l'enquête, leur statut parental ainsi que leur statut relationnel.

Analyses. Au total, 2443 femmes ont rempli le questionnaire et, afin de refléter les caractéristiques de la population à l'étude, 2202 d'entre elles ont été retenues après l'attribution d'un coefficient de pondération, calculé en utilisant les statistiques institutionnelles disponibles concernant le genre, la faculté, le cycle d'études et le statut (temps plein ou partiel). Cette procédure, utilisée pour corriger les biais d'échantillonnage, assure que les résultats soient généralisables à la population des étudiantes de l'UQAM. Sur ces 2202 répondantes, 1970 ont déclaré avoir été sexuellement actives au cours des deux années précédentes avec au moins un partenaire masculin. Sur ces 1970 répondantes, 20 ont été éliminées en raison de données manquantes concernant au moins une des variables sociodémographiques. Cela a porté le nombre de cas valides pour l'analyse à 1950 répondantes. De ces 1950 cas, 1812 ont fourni des données sur toutes les variables utilisées dans les analyses et constituent conséquemment l'échantillon final.

\section{L'Étude PIXEL : Portrait de la santé sexuelle des jeunes adultes au Québec}

Procédures. De mars 2013 à juillet 2014, 2973 jeunes adultes âgés de 17 à 29 ans ont été recrutés dans neuf régions du Québec à l'aide d'un échantillonnage probabiliste stratifié des établissements 
scolaires et de centres qui leur sont affiliés (38,0\% en cégeps, 31,0\% en centres de formation professionnelle ou centres d'éducation aux adultes, 22,9\% à l'université et 8,2\% en carrefours jeunesse emploi). Les participants ont fourni un consentement électronique avant de participer à PIXEL. Les données ont été recueillies à l'aide d'un questionnaire auto-administré par ordinateur sous la supervision d'agentes de recherche. Les réponses ont été entreposées sur un serveur sécurisé. Le rapport méthodologique et le questionnaire sont disponibles sur le site de PIXEL (https://www.inspq.qc.ca/espace-itss/pixel/sante-sexuelle-jeunes-adultes). Le comité d'éthique de la recherche de l'Agence de la santé et des services sociaux de Montréal (ASSSM) a agi à titre de comité éthique évaluateur. Des certificats d'éthique de I'UQAM ainsi que du CHUM ont aussi été obtenus, de même que dans certains établissements d'enseignement collégiaux et universitaires.

Instrument de mesure. L'étude comprend diverses mesures dans trois domaines : 1) État de santé sexuelle (ITSS, grossesses non planifiées, bien-être sexuel) ; 2) Comportements sexuels et facteurs associés (comportements à risque et de protection) ; 3) Accès aux services de santé sexuelle (dépistage d'ITS, contraception, vaccination contre le virus du papillome humain (VPH), dépistage du cancer du col de l'utérus). Les participant-e-s ont été interrogé-e-s sur leur âge, leur trajectoire migratoire (naissance à l'extérieur du Canada ou parents nés à l'extérieur du Canada), leur accès à un médecin de famille, leur lieu de résidence, leur fréquentation des institutions ou services religieux, leur revenu annuel, leur niveau de scolarité ainsi que leur statut relationnel.

Analyses. Aucune procédure de pondération n'a été utilisée pour corriger les biais d'échantillonnage puisque l'information sur les registres scolaires n'était pas accessible. Comme dans d'autres études similaires, des biais d'échantillonnage sont à prévoir, notamment une représentation inégale de l'ensemble des programmes disponibles au Québec. Aux fins de cet article, l'échantillon comprend les 921 femmes sexuellement actives inscrites au cégep $(n=514)$ ou à l'université $(n=407)$, dont le dernier partenaire sexuel était un homme.

\section{Analyses statistiques pour les deux enquêtes}

Certaines questions posées dans une des deux enquêtes ne l'étaient pas dans l'autre. Lorsque cela est jugé pertinent, ces données sont présentées, même si elles ne sont disponibles que pour l'une des enquêtes. Des analyses univariées ont été effectuées pour montrer le profil d'utilisation de méthodes contraceptives, des expériences sexuelles et des connaissances quant à la CU. Des analyses subséquentes (tests de chi-carré) ont été effectuées pour explorer les différences dans l'utilisation de la $\mathrm{CH}$ et du dispositif/système intra-utérin, du préservatif masculin et féminin, de la CU ou d' " aucune méthode ", selon les caractéristiques des répondantes: trajectoire migratoire, orientation sexuelle et statut relationnel. Les répondantes pour lesquelles des données étaient manquantes ont été exclues des analyses. Le seuil de signification statistique a été fixé à un alpha de 0,05 . Les analyses ont été menées avec le logiciel Statistical Package for the Social Sciences (SPSS 22.0).

\section{RÉSUltats}

\section{Caractéristiques des échantillons}

Ainsi qu'on peut le voir au tableau 1, les trois échantillons varient légèrement quant à l'âge moyen, à l'image de la progression académique type au Québec. Une répondante sur cinq est née à l'extérieur 
du Canada ou a au moins un parent né à l'extérieur du Canada. La majorité des cégépiennes recrutées par PIXEL ont déclaré vivre chez leurs parents ( $83,9 \%)$, tandis que c'était le cas pour moins de la moitié des universitaires (PIXEL : 45,2\% ; UQAM : $34,7 \%$ ). Un peu plus du tiers (38\%) des étudiantes de I'UQAM ont indiqué qu'elles étaient "occasionnellement ou régulièrement en situation financière difficile ". Du côté de PIXEL, 66,9 \% des cégépiennes et 46,2 \% des universitaires indiquaient avoir un revenu annuel total avant impôts de moins de $10000 \$$. Entre $7 \%$ et $11 \%$ des répondantes des deux études retenues aux fins de cet article se sont identifiées comme lesbiennes, bisexuelles ou en questionnement. Un peu plus de la moitié des répondantes ont indiqué être en couple au moment de la collecte de données : $62,8 \%$ des cégépiennes et $67,7 \%$ des universitaires recrutées par PIXEL, et $55 \%$ des étudiantes de l'UQAM, sans toutefois indiquer le sexe du ou de la partenaire. L'âge moyen à la première relation sexuelle vaginale était de 16 ans et le nombre moyen de partenaires sexuels au cours des 12 mois précédents variait entre 2,1 et 2,4 pour les répondantes recrutées par PIXEL et à I'UQAM.

Tableau 1. Caractéristiques sociodémographiques des répondantes et variables associées aux comportements sexuels chez les jeunes femmes actives sexuellement avec au moins un partenaire masculin

\begin{tabular}{|c|c|c|c|}
\hline Caractéristiques & $\begin{array}{l}\text { Cégeps-PIXEL } \\
(n=514)\end{array}$ & $\begin{array}{l}\text { Universités- } \\
\text { PIXEL } \\
(n=407)\end{array}$ & $\begin{array}{l}\text { UQAM-ESS } \\
(n=1812)\end{array}$ \\
\hline \multicolumn{4}{|l|}{ Données démographiques } \\
\hline Âge moyen & 17,1 ans $(1,7)$ & 22,0 ans $(2,3)$ & 24,8 ans $(5,5)$ \\
\hline $\begin{array}{l}\text { Trajectoire migratoire : née à } \\
\text { l'extérieur du Canada ou ayant un } \\
\text { parent né à l'extérieur du Canada }\end{array}$ & $18,9 \%$ & $17,0 \%$ & $21,0 \%$ \\
\hline $\begin{array}{l}\text { Lieu de résidence : habite chez ses } \\
\text { parents }\end{array}$ & $83,9 \%$ & $45,2 \%$ & $34,7 \%$ \\
\hline $\begin{array}{l}\text { Connaît des difficultés financières : } \\
\text { occasionnellement ou régulièrement }\end{array}$ & - & - & $38,3 \%$ \\
\hline $\begin{array}{l}\text { Revenu annuel avant impôt de moins } \\
\text { de } 10000 \$\end{array}$ & $66,9 \%$ & $46,2 \%$ & - \\
\hline $\begin{array}{l}\text { Présentement en relation de couple } \\
\text { Variables associées à la sexualité }\end{array}$ & $62,8 \%$ & $67,7 \%$ & $54,5 \%$ \\
\hline $\begin{array}{l}\text { Orientation sexuelle auto-identifiée : } \\
\text { autre qu'hétérosexuelle } \\
\text { Âge moyen à la première relation }\end{array}$ & $7,2 \%$ & $8,1 \%$ & $10,5 \%$ \\
\hline $\begin{array}{l}\text { sexuelle vaginale } \\
\text { Nombre moyen de partenaires sexuels }\end{array}$ & 16,0 ans $(1,5)$ & 16,5 ans $(1,9)$ & 16,7 ans $(2,3)$ \\
\hline $\begin{array}{l}\text { au cours des } 12 \text { derniers mois } \\
\text { A déjà eu un test de dépistage d'ITS }\end{array}$ & $2,0(2,2)$ & $2,2(3,4)$ & $2.3(4,1)$ \\
\hline au cours de la vie & $42,6 \%$ & $67,6 \%$ & $75,4 \%$ \\
\hline
\end{tabular}

Note : - = données non disponibles. 


\section{Grossesse, désir d'enfant et utilisation de méthodes contraceptives}

Grossesses antérieures et désir d'enfant. Un peu plus d'une cégépienne sur 20 (6\%) et $7 \%$ des universitaires recrutées par PIXEL ont déclaré avoir vécu une grossesse ou plus au cours de leur vie. Environ $1 \%$ des répondantes recrutées par PIXEL ont indiqué avoir un enfant, tandis qu'une étudiante de I'UQAM sur 10 est parent (10\%). Jusqu'à $86 \%$ des étudiantes de l'UQAM (incluant les femmes ayant déjà un enfant) ont mentionné souhaiter avoir un ou des enfants $(17,3 \%$ "probablement ", $63,8 \%$ « oui, mais plus tard », et 4,8\% « oui, maintenant »). En contrepartie, 7,6\% des étudiantes déclarent ne pas vouloir d'enfants et $6,4 \%$ ne se voient probablement pas devenir parents dans l'avenir. Ainsi, $95 \%$ des étudiantes de l'UQAM indiquent un besoin de contraception au moment de l'enquête (données non présentées).

Utilisation de contraception. La contraception hormonale $(\mathrm{CH})$ (pilule, timbre, anneau vaginal) est le type de contraception le plus populaire, suivie par le condom (méthode utilisée " actuellement » dans PIXEL ou «au cours des 24 derniers mois » dans ESS-UQAM) (tableau 2). L'utilisation du condom féminin est marginale (données non présentées). Le recours à la méthode du retrait (coït interrompu) est rapporté par une répondante sur cinq parmi les cégépiennes de PIXEL $(20 \%)$ et parmi les étudiantes de I'UQAM (20,9\%), tandis que c'est le cas d'une répondante sur sept parmi les universitaires de PIXEL (15\%). L'utilisation de la contraception intra-utérine (système intra-utérin hormonal au lévonorgestrel [SIU-LNG] ou dispositif intra-utérin de cuivre [DIU-cuivre]) a été rapportée par peu de répondantes de PIXEL (cégeps : 1,0\%; universités : $3,9 \%$ ) et une proportion plus importante de répondantes de I'UQAM (7,8\%). Parmi les étudiantes de l'UQAM, 2,9\% n'ont utilisé aucune méthode contraceptive au cours des deux dernières années, une proportion similaire à celle des universitaires recrutées par PIXEL (3,2\%). Cette proportion double chez les répondantes du cégep $(6 \%)$.

\section{Utilisation de contraception lors de la dernière relation sexuelle (PIXEL seulement)}

La double protection (condom $+\mathrm{CH}$ ) à la dernière relation sexuelle est rapportée par un cinquième des femmes actives sexuellement avec un partenaire de sexe masculin (cégep : 22,2\%; université : $23,2 \%)$. Sans que cela constitue une différence statistiquement significative, on relève que ce recours à la double protection est moins fréquent avec un partenaire avec lequel existe une relation de couple (cégep : 20,1\% ; université : $21,4 \%$ ) qu'avec un partenaire avec lequel n'existe pas une telle relation (cégep : $28,5 \%$; université : 28,8\%). En contrepartie, $13,0 \%$ des cégépiennes et 9,8\% des universitaires ont indiqué n'avoir utilisé ni condom, ni contraception régulière. Fait intéressant : 17,6\% des répondantes cégépiennes et $8,5 \%$ des répondantes universitaires ont indiqué avoir utilisé à la fois la $\mathrm{CH}$ et le retrait lors de leur dernière relation sexuelle. 
Tableau 2. Types de méthodes contraceptives utilisées chez les jeunes femmes sexuellement actives avec au moins un partenaire masculin

\begin{tabular}{llll}
\hline Méthodes contraceptives* & $\begin{array}{l}\text { Cégeps-PIXEL } \\
(\mathrm{n}=514)\end{array}$ & $\begin{array}{l}\text { Universités-PIXEL } \\
(\mathrm{n}=407)\end{array}$ & $\begin{array}{l}\text { UQAM-ESS } \\
(\mathrm{n}=1812)\end{array}$ \\
\hline & $\%$ (actuellement) & $\%$ (actuellement) & $\begin{array}{l}\% \text { (au cours des } 24 \\
\text { derniers mois) }\end{array}$ \\
Contraception hormonale & $77,1 \%$ & $80,0 \%$ & $74,8 \%$ \\
Préservatifs & $51,2 \%$ & $45,9 \%$ & $67,9 \%$ \\
Retrait (Coït interrompu) & $20,0 \%$ & $14,7 \%$ & $20,9 \%$ \\
DIU cuivre/ hormonal & $1,0 \%$ & $3,9 \%$ & $7,8 \%$ \\
Méthodes naturelles & $1,9 \%$ & $2,7 \%$ & $5,1 \%$ \\
Stérilisation & $\mathrm{nd}$ & $\mathrm{nd}$ & $1,7 \%$ \\
(féminine/masculine) & $1,4 \%$ & $1,2 \%$ & $1,2 \%$ \\
Contraception injectable & & & \\
& & $0,2 \%$ & $0,4 \%$ \\
Spermicide & $0,6 \%$ & $3,2 \%$ & $2,9 \%$ \\
Aucune méthode & $6,0 \%$ & &
\end{tabular}

*Possibilité d'indiquer plus d'une méthode.

Concernant l'endroit où la méthode contraceptive a été prescrite, 55,4 \% des cégépiennes et 62,8 \% des universitaires recrutées dans PIXEL ont indiqué avoir reçu la prescription en clinique de médecine familiale ou dans une clinique non spécialisée pour les ITS, alors que 23,2 \% des cégépiennes et 18,1\% des universitaires l'ont reçue dans une clinique jeunesse ou en CLSC. Seulement 7,2\% des cégépiennes et $3,9 \%$ des universitaires indiquent avoir reçu leur prescription au sein de leur établissement scolaire.

\section{La contraception d'urgence : recours et connaissances}

Recours à la contraception d'urgence et raisons évoquées. Parmi les répondantes de l'UQAM, près de la moitié ont déclaré avoir utilisé la CU dans leur vie (48,6\%). La plupart l'ont utilisée une fois (51,5\%), $26,3 \%$ deux fois, $11,6 \%$ trois fois et $10,6 \%$, quatre fois ou plus. Les principales raisons de cette utilisation évoquées par les étudiantes de l'UQAM étaient le bris d'un préservatif $(37,9 \%)$, l'oubli d'une pilule $(26,8 \%)$, le préservatif non disponible $(16,0 \%)$, le souhait de ne pas utiliser de préservatif (ellesmêmes ou leur partenaire, $12,4 \%)$ et un échec de la méthode contraceptive (6,9\%). Parmi les répondantes de PIXEL, $21,0 \%$ des étudiantes au cégep et 15,7\% des universitaires ont rapporté avoir utilisé la CU au cours des 12 derniers mois ; 7,4\% des cégépiennes et 2,6\% des universitaires l'ont utilisée plus d'une fois au cours de la dernière année.

Connaissances sur la contraception d'urgence. Seulement 3,3\% des étudiantes de l'UQAM déclaraient ne pas connaître l'existence de la CU. Le tiers (35,3\%) croyaient que la CU devait être prise dans les vingt-quatre heures suivant la relation sexuelle mal ou non protégée, alors que $96,1 \%$ ne savaient pas qu'elle pouvait être prise jusqu'à cinq jours après celle-ci. L'utilisation du DIU de cuivre d'urgence était peu connue chez les étudiantes, près des deux tiers $(64,2 \%)$ ignorant cette option. Dans PIXEL, seulement le tiers des étudiantes interrogées sur leurs connaissances liées à la CU ont répondu à l'énoncé " La CU n'est efficace que si elle est prise dans les 24 heures après avoir eu des rapports 
sexuels non protégés 》 en indiquant qu'il s'agissait d'une fausse affirmation (cégep: 32,4\%; université : 34,6\%). Toutefois, la question de l'accès à la CU semblait mieux connue puisque 86,2\% des cégépiennes et $85,9 \%$ des étudiantes universitaires soutenaient l'affirmation « La contraception orale d'urgence (pilule du lendemain) est disponible à la pharmacie directement, sans prescription du médecin ». 
Tableau 3. Différences entre les sous-groupes quant à l'utilisation des méthodes contraceptives hormonales, du condom, du coït interrompu, d'aucune méthode contraceptive et l'utilisation de méthodes contraceptives d'urgence (Tests khi-deux)*

\begin{tabular}{|c|c|c|c|c|c|c|c|c|c|}
\hline \multirow{2}{*}{$\begin{array}{l}\text { Utilisation } \\
\text { contraceptive } \\
\text { (\%) }\end{array}$} & \multicolumn{3}{|c|}{ Trajectoire migratoire } & \multicolumn{3}{|c|}{ Orientation sexuelle } & \multicolumn{3}{|c|}{ Statut relationnel } \\
\hline & $\begin{array}{l}\text { Née au } \\
\text { Canada de } \\
\text { parents nés } \\
\text { au Canada }\end{array}$ & $\begin{array}{l}\text { Née à l'extérieur } \\
\text { du Canada ou } \\
\text { parents nés à } \\
\text { l'extérieur du } \\
\text { Canada }\end{array}$ & & $\begin{array}{l}\text { Hétéro- } \\
\text { sexuelle }\end{array}$ & $\begin{array}{l}\text { Autre } \\
\text { qu'hétérosexuelle }^{1}\end{array}$ & & $\begin{array}{l}\text { Présentement } \\
\text { célibataire }\end{array}$ & $\begin{array}{l}\text { Présentemen } \\
\text { t en relation } \\
\text { de couple }\end{array}$ & \\
\hline \multicolumn{10}{|l|}{ Cégep PIXEL ${ }^{3}$} \\
\hline Préservatif & $33,9 \%$ & $35,8 \%$ & & $34,3 \%$ & $38,9 \%$ & & $44,4 \%$ & $31,3 \%$ & $\mathrm{p}<, 05^{*}$ \\
\hline $\begin{array}{l}\text { Contraception } \\
\text { hormonale }\end{array}$ & $81,1 \%$ & $47,2 \%$ & $\mathrm{p}<, 001^{\star * *}$ & $75,6 \%$ & $65,7 \%$ & & $67,7 \%$ & $77,3 \%$ & $\mathrm{p}<, 05^{\star}$ \\
\hline Aucune méthode & $13,1 \%$ & $43,8 \%$ & $\mathrm{p}<, 001^{\star \star \star}$ & $18,5 \%$ & $22,9 \%$ & & $24,2 \%$ & $17,0 \%$ & \\
\hline Coït interrompu & $5,0 \%$ & $9,0 \%$ & & $5,3 \%$ & $11,4 \%$ & & $6,5 \%$ & $5,5 \%$ & \\
\hline $\begin{array}{l}\text { Contraception } \\
\text { d'urgence } \\
\text { (dernière relation } \\
\text { sexuelle) }\end{array}$ & $2,0 \%$ & $9,4 \%$ & $\mathrm{p}<, 001^{\star * *}$ & $3,2 \%$ & $5,6 \%$ & & $5,2 \%$ & $2,7 \%$ & \\
\hline $\begin{array}{l}\text { Contraception } \\
\text { d'urgence (12 } \\
\text { mois) }\end{array}$ & $17,7 \%$ & $34,8 \%$ & $\mathrm{p}<, 001^{* * *}$ & $19,5 \%$ & $40,5 \%$ & $\mathrm{p}<, 005^{*}$ & $23,7 \%$ & $20,1 \%$ & \\
\hline \multicolumn{10}{|l|}{ Université PIXEL ${ }^{3}$} \\
\hline Préservatif & $33,9 \%$ & $43,5 \%$ & & $36,2 \%$ & $30,3 \%$ & & $49,5 \%$ & $31,0 \%$ & $\mathrm{p}<, 005^{*}$ \\
\hline $\begin{array}{l}\text { Contraception } \\
\text { hormonale }\end{array}$ & $80,8 \%$ & $68,2 \%$ & $\mathrm{p}<, 05^{\star}$ & $80,4 \%$ & $57,6 \%$ & $\mathrm{p}<, 005^{*}$ & $67,6 \%$ & $82,7 \%$ & $\mathrm{p}<, 005^{*}$ \\
\hline Aucune méthode & $13,8 \%$ & $22,7 \%$ & & $14,4 \%$ & $24,2 \%$ & & $23,8 \%$ & $11,9 \%$ & $\mathrm{p}<, 005^{*}$ \\
\hline Coït interrompu & $4,5 \%$ & $9,1 \%$ & & $4,9 \%$ & $12,1 \%$ & & $6,7 \%$ & $5,1 \%$ & \\
\hline $\begin{array}{l}\text { Contraception } \\
\text { d'urgence }\end{array}$ & $1,5 \%$ & $2,9 \%$ & & $1,9 \%$ & $0,0 \%$ & & $4,6 \%$ & $0,7 \%$ & $\mathrm{p}<, 05^{*}$ \\
\hline
\end{tabular}


(dernière relation

sexuelle)

Contraception

d'urgence (12

$29,2 \%$

$\mathrm{p}<, 005^{*}$

$15,4 \%$

$20,0 \%$

$25,7 \%$

$11,8 \%$

$p<, 005^{*}$

mois)

UQAM-ESS

\section{Préservatif}

$67,7 \% \quad 68,9 \%$

Contraception

hormonale

$75,7 \%$

$68,3 \%$

$\begin{array}{lll} & 67,5 \% & 70,6 \% \\ \mathrm{p}<, 05^{\star} & 76,3 \% & 62,0 \% \\ & 2,8 \% & 3,5 \% \\ \mathrm{p}<, 001^{\star * *} & 18,5 \% & 28,2 \%\end{array}$

$\begin{array}{ll}p<, 001^{* *} & 80,4 \% \\ \text { * } & 76,5 \%\end{array}$

$56,8 \%$

$\mathrm{p}<, 001^{\star * *}$

Aucune méthode

$3,1 \%$

$1,2 \%$

$18,3 \%$

$28,8 \%$

$28,2 \%$

$2,6 \%$

$73,4 \%$

$\mathrm{p}<, 001^{\star \star \star}$

Contraception

d'urgence

(période de réf. :

vie)

${ }^{1}$ Inclut les femmes qui s'identifient comme lesbiennes, bisexuelles et en questionnement.

${ }^{2}$ Inclut aussi les participantes qui indiquent, dans PIXEL, que le dernier partenaire était un partenaire de couple.

${ }^{3}$ Sauf avis contraire, les données issues de l'étude PIXEL présentées dans le tableau concernent la dernière relation sexuelle vaginale avec un homme ; les données tirées de ESS-UQAM portent sur les 24 derniers mois.

* Des analyses bivariées ont été réalisées sur l'ensemble des variables à l'étude, seuls les résultats statistiquement significatifs sont indiqués pour alléger le tableau. 


\section{La contraception : un choix modulé selon certaines caractéristiques ?}

Une variation des choix contraceptifs selon l'orientation sexuelle, le statut relationnel des étudiantes et le fait d'avoir ou non une trajectoire migratoire est observée (tableau 3). En ce qui a trait au facteur de la trajectoire migratoire, on constate que les universitaires de I'UQAM nées hors du Canada ou de parents nés hors du Canada sont moins nombreuses à utiliser la $\mathrm{CH}$ que celles qui sont nées au Canada et de parents nés au Canada (68\% c. $76 \%$, p < 0,05). Le retrait et l'utilisation de la contraception d'urgence (CU) sont plus répandus chez les femmes ayant une trajectoire migratoire que chez celles qui n'en signalent pas (28,8\% c. $18,3 \%$ et $61,3 \%$ c. $46,9 \% ; p<0,001)$. Dans PIXEL, une différence marquée est notée en ce qui a trait à l'utilisation de $\mathrm{CH}$ à la dernière relation sexuelle entre les étudiantes ne présentant pas de trajectoire migratoire et celles qui en ont une (cégep : 81,1\% c. 47,4\%, $p<0,001$; université : $80,8 \%$ c. $68,9 \%, p<0,05)$. Les étudiantes qui ont une trajectoire migratoire sont aussi plus nombreuses à indiquer n'avoir utilisé aucune méthode contraceptive lors de leur dernière relation sexuelle et avoir utilisé la CU dans les 12 derniers mois ou lors de leur dernière relation sexuelle que celles qui sont nées au Canada ou dont les parents sont nés au Canada.

Les choix contraceptifs diffèrent selon l'orientation sexuelle déclarée des étudiantes. Ainsi, l'utilisation de $\mathrm{CH}$ au cours des 24 derniers mois est moins répandue parmi les étudiantes de l'UQAM s'identifiant comme lesbiennes, bisexuelles et en questionnement comparativement aux étudiantes qui s'identifient comme hétérosexuelles (62\% c. $76 \% ; p<0,001)$. Le retrait est plus répandu chez les femmes qui s'identifient autrement que comme hétérosexuelles que chez les étudiantes qui s'identifient comme hétérosexuelles (28\% c. 18,5\%; $p<0,01$ ). Dans PIXEL, chez les cégépiennes, il n'y a pas de différence significative selon l'orientation sexuelle en ce qui concerne l'utilisation de $\mathrm{CH}$. À l'université, cette différence se révèle statistiquement significative : $80,4 \%$ des étudiantes qui s'identifient comme hétérosexuelles utilisent une $\mathrm{CH}$ par rapport à $57,6 \%$ de celles qui s'identifient autrement $(p<0,005)$. Les étudiantes recrutées par PIXEL qui s'identifient autrement que comme hétérosexuelles sont plus nombreuses à n'avoir utilisé aucune méthode contraceptive à la dernière relation sexuelle avec un partenaire masculin que celles qui se disent hétérosexuelles (cégépiennes: 22,9\% c. 18,5\%; universitaires : $24,2 \%$ c. $14,4 \%$ ), mais ces différences ne sont pas significatives.

Le statut relationnel, soit le fait d'être en couple, module le recours au condom. Parmi les étudiantes de I'UQAM, le condom masculin est significativement moins utilisé par celles qui déclarent être actuellement en couple que par celles qui s'identifient comme célibataires ou non engagées avec un partenaire (57\% c. $81 \%, p<0,001)$. Dans PIXEL, la même tendance est observée à la question portant sur la dernière relation sexuelle : les cégépiennes et les universitaires dont le partenaire à la dernière relation sexuelle était un partenaire « de couple » sont significativement moins nombreuses à avoir utilisé un condom lors de cette relation que celles dont le dernier partenaire n'était pas un partenaire « de couple » (cégépiennes : 31,3\% c. 44,4 \%, p < 0,05; universitaires : 31,0\% c. 49,5\%, p < 0,005). Les universitaires recrutées par PIXEL dont le dernier partenaire était un partenaire « de couple » sont aussi moins nombreuses à avoir eu recours à la CU dans les 12 derniers mois que celles dont le dernier partenaire n'était pas un partenaire « de couple » $(11,8 \%$ c. $25,7 \%, p<0,005)$. 


\section{Discussion}

Les résultats de ces deux enquêtes montrent qu'en général au Québec, la proportion d'étudiantes utilisant une méthode contraceptive efficace (Trussell, 2011) est élevée et que, parallèlement, celle des étudiantes n'utilisant aucune contraception est plutôt faible, soit en deçà de 6 \%. La double protection, soit la combinaison du préservatif et d'une autre méthode contraceptive lors de la même relation sexuelle, n'est exercée que par une répondante sur cinq, ce qui est semblable à ce que rapportent les écrits traitant de ce sujet (Higgins et al., 2014 ; Hood et al., 2014 ; Pazol, Kramer et Hogue, 2010 ; Santelli et al., 1997 ; Tyler et al., 2014). Les données de PIXEL apportent toutefois une précision supplémentaire, à savoir que cette proportion est plus importante chez les répondantes dont le dernier partenaire n'est pas un partenaire de couple (28\%).

Pour ce qui est des méthodes utilisées au cours des 12 derniers mois (PIXEL) ou des 24 derniers mois (ESS-UQAM), il ressort que la $\mathrm{CH}$, en particulier la pilule contraceptive, est la méthode la plus utilisée, et ce, par les trois quarts des répondantes, suivie de l'utilisation du condom. Ce niveau d'utilisation de la pilule contraceptive et du condom dénote une certaine tendance à se conformer à la " norme contraceptive » chez les jeunes adultes, lesquels explorent peu les autres options qui leur sont accessibles (Charton et Lapierre-Adamcyk, 2008). Au Québec, une augmentation notable de l'utilisation de la pilule contraceptive chez les femmes de moins de 20 ans entre 2003 et 2009-2010 est rapportée (Joubert et Du Mays, 2014). La pilule contraceptive est par ailleurs la méthode contraceptive la plus utilisée aux États-Unis (Daniels et al., 2015 ; Jones, Mosher et Daniels, 2012 ; Mosher et Jones, 2010), en France (Bajos et al., 2012 ; Jost et al., 2014) et dans plusieurs pays industrialisés (Black et al., 2009 ; Darroch, 2013). De façon contrastée, selon l'Enquête sur la santé dans les collectivités canadiennes de 2003 et 2009-2010 au Québec (Joubert et Du Mays, 2014), le National Survey of Contraceptive Use Among Canadian Women of Reproductive Age (2006) (Black et al., 2009), ainsi que d'autres données canadiennes (Rotermann, 2012 ; Rotermann, Dunn et Black, 2015), le condom apparaît comme la méthode contraceptive la plus utilisée par les jeunes femmes, en particulier par celles qui ont moins de 20 ans. La majorité des enquêtes indiquent cependant que la fréquence de l'utilisation du condom décroît avec l'âge et que la méthode contraceptive la plus utilisée chez les femmes de 20-24 ans ou de 20-29 ans est la pilule contraceptive (Black et al., 2009 ; Jones et al., 2012 ; Joubert et Du Mays, 2014 ; Rotermann, 2012).

Malgré les mesures mises en place pour faciliter l'accès à la $\mathrm{CH}$ et la grande disponibilité des condoms, la troisième méthode la plus utilisée par les étudiantes sondées est le coït interrompu : $20 \%$ des cégépiennes et $15 \%$ des universitaires de l'étude PIXEL ainsi que 20,9\% des étudiantes de l'UQAM y ont recours. Le même constat émerge de l'Enquête canadienne sur la contraception (Black et al., 2009) : 17,3 \% des Canadiennes de 15-19 ans et $12 \%$ des Canadiennes de 20-29 ans ont recours au coït interrompu. Une analyse spécifique de l'utilisation de cette méthode dans le National Survey of Family Growth 2006-2010 (Higgins et Wang, 2015) a montré que, chez les 15-24 ans, $14 \%$ des Américaines avaient utilisé cette méthode lors de leur dernière relation sexuelle, $7 \%$ rapportant ne se fier qu'à cette méthode pour leur contraception. Fait intéressant, $65 \%$ des femmes utilisaient le coït interrompu conjointement avec une méthode contraceptive très efficace et parfois même avec le condom, une tendance aussi observée dans les données de PIXEL, quoique de façon plus marginale. Les prochaines études québécoises et canadiennes devraient viser une meilleure compréhension de l'utilisation du coït interrompu à titre de méthode contraceptive unique ou d'appoint. 
La proportion d'étudiantes affirmant n'utiliser aucune méthode contraceptive " actuellement » était de $6,0 \%$ (cégeps) et 3,2\% (universités) dans PIXEL, et de 2,9\% dans l'étude de l'UQAM. Au Canada, I'ESCC 2009-2010 (Norman et al., 2013) montre que 15,4\% des Canadiens âgés de 15 à 24 ans souhaitant éviter une grossesse n'avaient pas utilisé de contraception lors de leur dernière relation sexuelle ; cette proportion étant de $28 \%$ dans les Territoires, $20 \%$ en Colombie-Britannique et en Ontario, $13 \%$ à $17 \%$ dans les provinces de l'Atlantique et les Prairies et $7 \%$ au Québec. Ce faible taux de non-utilisation de la contraception lors de la dernière relation sexuelle rapporté au Québec pourrait être en lien avec le coût abordable des contraceptifs au Québec, la facilité d'accès aux cliniques jeunesse, scolaires et de planification des naissances (Henshaw et Jones, 1988), et l'implantation de l'ordonnance collective de contraception hormonale et du stérilet dans les cliniques jeunesse et scolaires du Québec en 2007 (Guilbert et al., 2013 ; Guilbert et al., 2014), utilisé jusqu'à la fin mars 2018 et progressivement remplacé par les activités liées l'approbation de droits de prescription de la contraception pour les infirmières survenues en 2014 (Guilbert et al., 2016). Les données de PIXEL confirment d'ailleurs qu'une cégépienne sur trois $(30,4 \%)$ et une universitaire sur cinq $(22,0 \%)$ obtiennent leurs prescriptions de contraceptifs d'une clinique jeunesse en CLSC ou en milieu scolaire. Cet accès rapide aux contraceptifs dans les milieux de vie des jeunes vient renforcer les services offerts par les cliniques médicales et les cliniques de médecine familiale qui demeurent des lieux de prescription de la contraception pour les deux tiers des jeunes femmes sondées dans PIXEL.

Force est d'admettre, toutefois, que les jeunes femmes ne bénéficient pas toutes de la même couverture contraceptive. Dans les deux enquêtes, la trajectoire migratoire et l'orientation sexuelle semblent influer sur l'usage d'une contraception hormonale régulière, qui est plus répandue chez les jeunes femmes qui sont nées au Canada de parents nés au Canada et chez celles qui se disent hétérosexuelles. De telles disparités sont observées de façon plus marquée aux États-Unis, qu'il soit question de la trajectoire migratoire (Dehlendorf et al., 2010) ou de l'orientation sexuelle (Charlton et al., 2013). Bien que les relations sexuelles entre partenaires du même sexe ne présentent pas de risque reproductif, plusieurs jeunes femmes des deux études s'identifient comme étant en questionnement ou bisexuelles et sont par là également concernées par le sujet. De plus, la littérature démontre que l'orientation sexuelle autorapportée et les comportements sexuels peuvent être divergents (Copen, Chandra et FeboVazquez, 2016 ; Igartua et Montoro, 2015). Plusieurs personnes pourraient ainsi s'identifier comme homosexuelles, mais avoir des relations sexuelles avec des personnes de sexe différent. La période développementale de l'adulte émergent peut aussi être une occasion d'expérimenter différents comportements sexuels, avec des partenaires variés (Katz-Wise, 2014). Ces résultats laissent croire que la mise en place d'initiatives visant à faciliter l'accès à la contraception au Québec (Guilbert et al., 2013) n'atteint pas uniformément toutes les jeunes femmes. Tel qu'en a fait la recommandation le Consensus canadien sur la contraception (Black et al., 2015a), il apparaît nécessaire d'améliorer l'accès aux services de façon à assurer que toutes les Canadiennes soient en mesure de planifier et d'espacer leurs grossesses et d'atteindre leurs objectifs génésiques. Une attention particulière devra être portée à l'adaptation des services contraceptifs aux femmes en situation de vulnérabilité ou de stigmatisation. L'évaluation subjective que fait la personne de sa situation financière, bien que reconnue comme un indicateur fiable lors d'enquêtes auprès de populations similaires (Ministère de l'Enseignement supérieur et de la Recherche (France), 2011), peut être complexifiée si elle cible des étudiantes pouvant potentiellement s'appuyer sur d'autres sources de revenus comme le salaire des parents si elles sont à charge. Cela est principalement le cas pour les cégépiennes et une proportion importante d'étudiantes 
universitaires. Pour ces raisons, l'évaluation de sa situation socioéconomique par la répondante n'a pas été retenue dans les présentes analyses, mais mériterait une attention soutenue lors d'études futures.

À l'instar de ce qu'ont constaté plusieurs études ayant exploré les liens entre le statut relationnel et l'usage d'une contraception régulière (Manlove et al., 2014), les étudiantes en couple ou dont le dernier partenaire sexuel était un partenaire de couple ont davantage recours à la $\mathrm{CH}$ que les étudiantes célibataires ou dont le dernier partenaire sexuel était un partenaire autre que de couple. Cette hausse de l'usage de la $\mathrm{CH}$ se fait au détriment de l'usage du condom. Des efforts de sensibilisation ciblés pourraient améliorer le recours à une protection permettant à la fois de prévenir les grossesses non désirées et la transmission des ITS. À ce sujet, les données obtenues par l'étude PIXEL sur la double protection montrent que seulement une étudiante sur trois indique avoir opté pour la double protection à la dernière relation sexuelle. Tant au Québec qu'à l'étranger, c'est une pratique qui ne semble pas ancrée dans les habitudes, malgré la recrudescence des cas déclarés d'ITS chez les jeunes adultes (Blouin, Venne et Lambert, 2016). Dans l'étude de Tyler et ses collègues (2014), 20,7\% des adolescentes et jeunes adultes ont eu recours à la double protection lors de leur dernière relation sexuelle, un taux plus élevé que dans l'étude d'Eisenberg et ses collègues (2012) menée auprès de 5178 femmes âgées de 15 à 44 ans (7,3\%). Cette dernière étude relève toutefois un recours à la double protection plus fréquent chez les jeunes adultes, soit $22,8 \%$ des $15-20$ ans et $12 \%$ des $21-25$ ans, que chez les femmes âgées de plus de 25 ans (Eisenberg et al., 2012).

Selon PIXEL et l'ESS-UQAM, une majorité de répondantes connaissaient bien l'existence de la CU, mais beaucoup moins son délai d'utilisation. Par ailleurs, bien que les connaissances sur le délai et l'utilisation correcte de la contraception d'urgence soient faibles, on constate que son utilisation par les jeunes femmes interrogées est importante. Ainsi, les deux tiers des étudiantes de l'UQAM ne connaissaient pas l'utilisation du stérilet au cuivre pour la CU. Une autre étude effectuée auprès de 627 étudiants universitaires a montré qu'il existe un manque de compréhension en ce qui a trait au délai d'utilisation, un manque de connaissances sur l'accès et la disponibilité, une confusion sur l'action de la CU et un manque d'information sur la période la plus fertile du cycle menstruel (Calabretto, 2009). Une autre étude révèle que $2 / 3$ de l'échantillon composé de 693 étudiants universitaires ont répondu de manière incorrecte ou ne connaissaient pas le délai d'utilisation pour la CU (Sawyer et Thompson, 2003). Selon l'étude d'Isley et ses collègues (2010), améliorer les connaissances sur les méthodes contraceptives et dissiper les perceptions erronées sur les méthodes et leur utilisation pourrait influer positivement sur le comportement des jeunes adultes. À la lumière de nos résultats, notons qu'il existe un besoin d'informations sur les modes d'action, les délais associés et la disponibilité de la CU chez les cégépiennes et les étudiantes universitaires.

L'utilisation de la CU dans les 12 derniers mois était rapportée par 21,2\% des cégépiennes et 15,2 \% des universitaires de PIXEL, tandis que 48,6 \% des étudiantes de I'UQAM rapportaient y avoir recouru au moins une fois dans leur vie. Selon les études, les résultats diffèrent quant au recours à la CU par les jeunes femmes : 1,6 \% (Virjo et Virtala, 2003), 2,3 \% (Black et al., 2006), 10 \% (Sorhaindo et al., 2002), $17 \%$ (Miller, 2011) et $65 \%$ (Free et Ogden, 2005). Les taux élevés de recours à la CU observés au Québec pourraient être attribuables à sa grande accessibilité. Depuis 2001, le pharmacien peut prescrire la $\mathrm{CU}$, alors que cet acte était réservé aux médecins antérieurement. D'un point de vue de santé publique, les efforts en prévention des grossesses non désirées ne doivent toutefois pas être restreints à la CU. Alors qu'il est connu qu'un plus grand accès à la CU augmente son utilisation, les 
études portant sur l'accessibilité de la CU montrent que cela ne réduit pas le nombre de grossesses non planifiées ni le taux d'IVG (Raymond, Trussell et Polis, 2007). La remise d'une CU constitue toutefois une excellente occasion pour les pharmaciens et les infirmières d'amener leur cliente à réfléchir à l'adoption d'une contraception régulière, puisque ces professionnels ont récemment obtenu de tels droits de prescription au Québec (Guilbert et al., 2016), et peuvent ainsi prévenir plus efficacement les grossesses non désirées.

\section{Forces et limites}

Les deux études sur lesquelles s'appuie cet article sont complémentaires et permettent de bien documenter les connaissances sur les méthodes contraceptives, y compris la contraception d'urgence, et leurs utilisations, chez les jeunes femmes québécoises adultes fréquentant un établissement d'éducation postsecondaire. Toutefois, les périodes de référence dans les deux études diffèrent, Pixel présentant des données se rapportant à l'utilisation de méthodes contraceptives dans les 12 mois précédents ou lors de la dernière relation sexuelle, alors que l'ESS-UQAM rapporte des comportements s'étant manifestés au cours des 24 mois précédents. Cette différence limite la comparaison directe entre les deux études. De même, certains thèmes sont présents dans une étude sans l'être dans l'autre, réduisant aussi la comparaison. Toutefois, les observations concernant l'âge aux premières relations sexuelles, l'orientation sexuelle, le statut relationnel, les méthodes contraceptives utilisées ainsi que les connaissances sur la contraception d'urgence sont similaires dans les deux études rapportées ici, ce qui accroît la validité de celles-ci. D'autres limites associées à l'interprétation des résultats doivent aussi être mentionnées, notamment les liens proposés entre les stratégies québécoises implantées dans les dernières années et les comportements contraceptifs des étudiantes interrogées. Sans mesure sériée avant et après la mise en place de telles stratégies, les résultats doivent être interprétés comme des hypothèses d'efficacité des mesures de prévention. Cette limite met en exergue la nécessité d'enquêtes régulières pour monitorer le comportement contraceptif, afin d'évaluer la portée des actions déployées. La nature exploratoire des analyses bivariées réalisées pour documenter l'usage contraceptif selon la trajectoire migratoire, l'orientation sexuelle et le statut relationnel restreint la portée des résultats. Des analyses plus poussées sont nécessaires pour mieux comprendre l'effet des inégalités sociales sur l'accès à la contraception. Enfin, d'autres biais peuvent être liés à la difficulté de se remémorer certains événements ou à la désirabilité sociale. Cela dit, la combinaison de deux approches, soit l'analyse des comportements contraceptifs adoptés dans les 12/24 derniers mois et celle des comportements adoptés dans le contexte de la dernière relation sexuelle avec un partenaire masculin, a permis de pallier certains de ces biais. Enfin, l'échantillon était composé de jeunes femmes inscrites au cégep ou à l'université et les résultats ne peuvent être généralisés à d'autres populations, notamment des populations en situation de vulnérabilité et potentiellement plus à risque de vivre des grossesses non planifiées.

\section{Conclusion}

Depuis de nombreuses années, la prévention des grossesses non planifiées est un objectif de santé publique. Différentes mesures ont été déployées pour atteindre cet objectif, dont l'accès facilité à la contraception hormonale et à la contraception d'urgence et la création de cliniques jeunesse. Ces différentes mesures doivent être maintenues et rehaussées pour augmenter l'usage de méthodes 
contraceptives efficaces chez toutes les jeunes femmes qui le désirent, peu importe la trajectoire migratoire ou l'orientation sexuelle. En amont, un projet pilote d'éducation à la sexualité à l'intention des enfants et des adolescents est actuellement à l'essai dans plusieurs écoles du Québec, et l'éducation à la sexualité sera intégrée au cheminement scolaire d'une façon formelle dès septembre 2018 (Gouvernement du Québec, 2017 ; Ministère de l'Éducation et de l'Enseignement supérieur, 2017). Outre cette initiative, de telles stratégies éducatives ne devraient pas se limiter à la période de l'enfance et de l'adolescence, puisque la population des jeunes adultes exprime des besoins quant aux connaissances sur la contraception. Des recherches plus poussées sur les connaissances et les besoins des jeunes adultes semblent pertinentes, tout comme un suivi régulier de l'évolution des comportements contraceptifs et des grossesses non désirées.

\section{Financement}

L'Enquête sur la santé sexuelle des étudiants de l'UQAM a été financée par une subvention de l'Institut Santé et société de I'UQAM. L'étude PIXEL a été financée par la Direction générale de la santé publique du ministère de la Santé et des Services sociaux (par le Service de lutte contre les infections transmissibles sexuellement et par le sang pour le volet général et par la Direction de la protection pour le volet VPH).

\section{Conflits d'intérêts}

Sylvie Lévesque et Sara Mathieu déclarent l'absence de conflit d'intérêts. Édith Guilbert informe qu'elle a participé aux comités consultatifs des compagnies pharmaceutiques suivantes durant les deux dernières années : Bayer Canada Itd, Allergan Canada Itd et Mithra Pharmaceuticals Itd.

LÉVESQUE, Sylvie, Ph.D.

Département de sexologie Université du Québec à Montréal,

MATHIEU-C., Sara., Ph.D.(c) Faculté des sciences de l'éducation Université de Montréal,

GUILBERT, Édith, M.D., M.Sc. Institut national de santé publique du Québec

LAMBERT, Gilles, M.D. Direction régionale de santé publique de Montréal

Équipe ESS-UQAM : BEAULIEU PRÉVOST, Dominic, Ph.D., BLAIS, Martin, Ph.D., BOISLARD, Marie-Aude, Ph.D., LÉVY, Joseph, Ph.D. Département de sexologie

UQAM 


\section{RÉFÉRENCES}

Agence de santé publique du Canada (2009), Ce que disent les mères : l'Enquête canadienne sur l'expérience de la maternité, Ottawa, 246 pages.

Bajos, N., Bohet, A., Le Guen, M., et Moreau, C. (2012). La contraception en France : nouveau contexte, nouvelles pratiques? Population et sociétés, (492).

Black, K. I., Mercer, C. H., Johnson, A. M., et Wellings, K. (2006). Sociodemographic and sexual health profile of users of emergency hormonal contraception: Data from a British probability sample survey. Contraception, 74(4), 309-312.

Black, A., Yang, Q., Wen, S. W., Lalonde, A. B., Guilbert, É., et Fisher, W. (2009). Contraceptive use among Canadian women of reproductive age: Results of a national survey. Journal of Obstetrics and Gynaecology Canada, 31(07), 627-640.

Black, A., Guilbert, É., Costescu, D., Dunn, S., Fisher, W., Kives, S., .. Todd, N. (2015a). Consensus canadien sur la contraception. Journal of Obstetrics and Gynaecology Canada, 37(11), 1036-1039. doi.org/10.1016/S1701-2163(16)30056-1

Black, A., Guilbert, É., Hassan, F., Chatziheofilou, I., Lowin, J., Jeddi, M., Filonenko, A., et Trussell, J. (2015b). The cost of unintended pregnancies in Canada: Estimating direct cost, role of imperfect adherence, and the potential impact of increased use of long-acting reversible contraceptives. Journal of Obstetrics and Gynaecology Canada, 37(12), 1086-1097.

Blouin, K., Venne, S., et Lambert, G. (2016). Portrait des infections transmissibles sexuellement et par le sang (ITSS) au Québec : année 2015 (et projections 2016). Gouvernement du Québec.

Calabretto, H. (2009). Emergency contraception-knowledge and attitudes in a group of Australian university students. Australian and New Zealand Journal of Public Health, 33(03), 234-239.

Centers for Disease Control and Prevention (CDC). (2012). Sexual experience and contraceptive use among female teens - United States, 1995, 2002, and 2006-2010. Morbidity and Mortality Weekly Report, 61(17), 297-301.

Charlton, B. M., Corliss, H. L., Missmer, S. A., Rosario, M., Spiegelman, D., et Austin, S. B. (2013). Sexual orientation differences in teen pregnancy and hormonal contraceptive use: An examination across 2 generations. American Journal of Obstetrics and Gynecology, 209(3), 204.e201-204.e208.

Charton, L., et Lapierre-Adamcyk, E. (2008). Systèmes de valeurs et méthodes contraceptives : mise en perspective à partir du recours à la stérilisation contraceptive en France et au Québec. Communication présentée au Colloque international de l'Aidelf, Québec, 25-29 août.

Copen, C., Chandra, A., et Febo-Vazquez, I. (2016). Sexual behavior, sexual attraction, and sexual orientation among adults aged 18-44 in the United States: Data from the 2011-2013 National Survey of Family Growth, National Health Statistics Reports, 88. 
Daniels, K., Daugherty, J., Jones, J., et Mosher, W. (2015). Current contraceptive use and variation by selected characteristics among women aged 15-44: United States, 2011-2013. National Health Statistics Reports, 86, 1-14.

Darroch, J. E. (2013). Trends in contraceptive use. Contraception, 87(03), 259-263.

Dehlendorf, C., Rodriguez, M. I., Levy, K., Borrero, S., et Steinauer, J. (2010). Disparities in family planning. American Journal of Obstetrics and Gynecology, 202(03), 214-220.

Eisenberg, D. L., Allsworth, J. E., Zhao, Q., et Peipert, J. F. (2012). Correlates of dual-method contraceptive use: An analysis of the National Survey of Family Growth (2006-2008). Infectious Diseases in Obstetrics and Gynecology, 2012, 1-6.

Free, C., et Ogden, J. (2005). Emergency contraception use and non-use in young women: The application of a contextual and dynamic model. British Journal of Health Psychology, 10(02), 237253.

Gouvernement du Québec. (2017). Éducation à la sexualité obligatoire dès la rentrée. Portail Québec. http://www.fil-information.gouv.qc.ca/Pages/Article.aspx?idArticle=2512145860 (14 décembre 2017)

Guilbert, É., Robitaille, J., Guilbert, A. C., Morin, D., et le Comité d'experts en planning familial de I'Institut national de santé publique du Québec. (2013). Challenges of implementing task-shifting in contraceptive care - an experience in Quebec, Canada. Contraception, 88, 587-590.

Guilbert, É. R., Robitaille, J., Guilbert, A. C., et Morin, D. (2014). Determinants of the implementation of a new practice in hormonal contraception by Quebec nurses. The Canadian Journal of Human Sexuality, 23(01), 34-48.

Guilbert, É., Wagner, M.-S., Bérubé, J., et Dubé, P.-A. (2016). Protocole de contraception du Québec. Gouvernement du Québec.

Hartnett, C. S., Lindley, L. L., et Walsemann, K. M. (2017). Congruence across sexual orientation dimensions and risk for unintended pregnancy among adult US women. Women's Health Issues, 27(02), 145-151.

Henshaw, S. K., et Jones, E. F. (1988). The delivery of family planning services in Ontario and Quebec. Family Planning Perspectives, 20(02), 80-87.

Higgins, J. A., Smith, N. K., Sanders, S. A., Schick, V., Herbenick, D., Reece, M., Dodge, B., et Fortenberry, J. D. (2014). Dual method use at last sexual encounter: A nationally representative, episode-level analysis of US men and women. Contraception, 90(04), 399-406.

Higgins, J. A., et Wang, Y. (2015). Which young adults are most likely to use withdrawal? The importance of pregnancy attitudes and sexual pleasure. Contraception, 91(04), 320-327. 
Hood, J. E., Hogben, M., Chartier, M., Bolan, G., et Bauer, H. (2014). Dual contraceptive use among adolescents and young adults: Correlates and implications for condom use and sexually transmitted infection outcomes. Journal of Family Planning and Reproductive Health Care, 40(03), 200-207.

Igartua, K. J., et Montoro, R. (2015). Les minorités sexuelles : concepts, prémisses et structure d'une approche clinique adaptée. Santé mentale au Québec, 40(3). doi :10.7202/1034909ar

Institut de la statistique du Québec. (2017a). Taux d'interruption volontaire de grossesse, d'hystérectomie et de stérilisation par groupe d'âge, Québec, 1976-2014. Gouvernement du Québec.

Institut de la statistique du Québec. (2017b). Taux de fécondité selon le groupe d'âge de la mère, indice synthétique de fécondité et âge moyen à la maternité, Québec, 1951-2016. Gouvernement du Québec.

Isley, M. M., Edelman, A., Kaneshiro, B., Peters, D., Nichols, M. D., Jensen, J. T. (2010). Sex education and contraceptive use at coital debut in the United States: Results from Cycle 6 of the National Survey of Family Growth. Contraception, 82, 236-242.

Janevic, T., Pallas, W. S., Ismayilova, L., et Bradley, H. E. (2012). Individual and community level socioeconomic inequalities in contraceptive use in 10 Newly Independent States: A multilevel cross-sectional analysis. International Journal for Equity in Health, 11(01), 69.

Jones, J., Mosher, W., et Daniels, K. (2012). Current contraceptive use in the United States, 20062010, and changes in patterns of use since 1995. National Health Statistics Reports, 60, 1-25.

Jost, S., Le Tohic, A., Chis, C., This, P., Grosdemouge, I., et Panel, P. (2014). Contraception des femmes françaises de 15 à 45 ans : enquête nationale sur un échantillon représentatif de 5963 femmes. Gynécologie Obstétrique et Fertilité, 42(06), 415-421.

Joubert, K., et Du Mays, D. (2014). Relations sexuelles et contraception : un portrait des jeunes au cours des années 2000. Zoom santé, 45, Institut de la statistique du Québec, 12 p.

Katz-Wise, S. L. (2014). Sexual fluidity in young adult women and men: Associations with sexual orientation and sexual identity development. Psychology and Sexuality, 6(2), 189-208. doi :10.1080/19419899.2013.876445

LegisQuébec. (2002 ; mis à jour 2017). Loi sur l'assurance-médicaments. Chapitre A29.01, 38 p. http://legisquebec.gouv.qc.ca/fr/pdf/cs/A-29.01.pdf

Manlove, J., Welti, K., Wildsmith, E., et Barry, M. (2014). Relationship types and contraceptive use within young adult dating relationships. Perspectives on Sexual and Reproductive Health, 46(1), 41-50. 
Miller, L. M. (2011). College student knowledge and attitudes toward emergency contraception. Contraception, 83(01), 68-73.

Ministère de l'Éducation et de l'Enseignement supérieur. (2017). Éducation à la sexualité. Parent et tuteurs. Gouvernement du Québec. http://www.education.gouv.qc.ca/parents-et-tuteurs/education$\underline{\text { a-la-sexualite/ }}$

Ministère de l'Enseignement supérieur et de la Recherche (France). (2011). La santé des étudiants 2011: Rapports. Paris : Ministère de l'Enseignement supérieur et de la Recherche, 95 p.

Mosher, W., et Jones, J. (2010). Use of contraception in the United States: 1982-2008. Vital and Health Statistics. Series 23, Data from the National Survey of Family Growth, 29,1-44.

Norman, W. V., Leung, V., Nuernberger, K., Dunn, S., et Soon, J. (2013). Sexually active youth in Canada: Regional variations in pregnancy risk, from the 2009-2010 Canadian Community Health Survey. Contraception, 88(03), 451.

Pazol, K., Kramer, M. R., et Hogue, C. J. (2010). Condoms for dual protection: Patterns of use with highly effective contraceptive methods. Public Health Reports, 125(02), 208-217.

Prentice, M., Storin, C., et Robinson, G. (2012). Make it Personal. How Pregnancy Planning and Prevention Help Students Complete College. Washington, DC : American Association of Community Colleges.

Raymond, E. G., Trussell, J., et Polis, C. B. (2007). Population effect of increased access to emergency contraceptive pills: A systematic review. Obstetrics \& Gynecology, 109(01), 181-188.

Rotermann, M., et McKay, A. (2009). Condom use at last sexual intercourse among unmarried, not living common-law 20- to 34-year-old Canadian young adults. Canadian Journal of Human Sexuality, 18(03), 75-87.

Rotermann, M. (2012). Sexual behaviour and condom use of 15- to 24-year-olds in 2003 and 2009/2010. Health Reports, 23(01), 41-45.

Rotermann, M., Dunn, S., et Black, A. (2015). Oral contraceptive use among women aged 15 to 49 : Results from the Canadian Health Measures Survey. Health Reports, 26(10), 21.

Santelli, J. S., Warren, C. W., Lowry, R., Sogolow, E., Collins, J., Kann, L., Kaufmann, B. R., et Celentano, D. D. (1997). The use of condoms with other contraceptive methods among young men and women. Family Planning Perspectives, 29(06), 261-267.

Sawyer, R. G., et Thompson, E. (2003). Knowledge and attitudes about emergency contraception in university students. College Student Journal, 37(4), 523-531. 
Sorhaindo, A., Becker, D., Fletcher, H., et Garcia, S. G. (2002). Emergency contraception among university students in Kingston, Jamaica: A survey of knowledge, attitudes, and practices. Contraception, 66(4), 261-268.

Trussell, J. (2011). Contraceptive failure in the United States. Contraception, 83(5), 397-404.

Tyden, T., Svanberg, A. S., Karlström, P.-O., et Lampic, C. (2006). Female university students' attitudes to future motherhood and their understanding about fertility. The European Journal of Contraception and Reproductive Health Care, 11(3), 181-189.

Tyler, C. P., Whiteman, M. K., Kraft, J. M., Zapata, L. B., Hillis, S. D., Curtis, K. M., Anderson, J., Pazol, K., et Marchbanks, P. A. (2014). Dual use of condoms with other contraceptive methods among adolescents and young women in the United States. Journal of Adolescent Health, 54(2), 169-175.

Virjo, I., et Virtala, A. (2003). Why do university students use hormonal emergency contraception? The European Journal of Contraception \& Reproductive Health Care, 8(3), 139-144.

Virtala, A., Kunttu, K., Huttunen, T., et Virjo, I. (2006). Childbearing and the desire to have children among university students in Finland. Acta Obstetricia et Gynecologica, 85, 312-316 